\title{
Nonlinear radial oscillations of neutron stars
}

\author{
Michael Gabler, ${ }^{1,2}$ Ulrich Sperhake, ${ }^{1,3}$ and Nils Andersson ${ }^{4}$ \\ ${ }^{1}$ Theoretisch-Physikalisches Institut, Friedrich-Schiller-Universität, Max-Wien-Platz 1, 07743 Jena, Germany \\ ${ }^{2}$ Max-Planck-Institut für Astrophysik, Karl-Schwarzschild-Str. 1, 85741 Garching, Germany \\ ${ }^{3}$ Theoretical Astrophysics 35017, California Institute of Technology, Pasadena, California 91125, USA \\ ${ }^{4}$ School of Mathematics, University of Southampton, Southampton, SO17 1BJ, United Kingdom
}

(Received 17 June 2009; published 9 September 2009)

\begin{abstract}
The effects of nonlinear oscillations in compact stars are attracting considerable current interest. In order to study such phenomena in the framework of fully nonlinear general relativity, highly accurate numerical studies are required. A numerical scheme specifically tailored for such a study is based on formulating the time evolution in terms of deviations from a stationary equilibrium configuration. Using this technique, we investigate over a wide range of amplitudes nonlinear effects in the evolution of radial oscillations of neutron stars. In particular, we discuss mode coupling due to nonlinear interaction, the occurrence of resonance phenomena, shock formation near the stellar surface as well as the capacity of nonlinearities to stabilize perturbatively unstable neutron star models.
\end{abstract}

DOI: 10.1103/PhysRevD.80.064012

PACS numbers: 04.25.D-, 04.40.Dg, 97.10.Sj

\section{INTRODUCTION}

The observational and theoretical study of stellar oscillations aimed at gaining insight into stellar structure, an area called asteroseismology, has been a rich research field for a long time. This includes the period-luminosity relation for Cepheids and the variability of RR Lyrae stars first discovered by Leavitt and Pickering and Flemming, respectively, [1,2]; see [3-5] and references therein for more recent studies. Similarly, much insight into the structure of the Sun has been obtained via helioseismology (see [6] for a recent review). Oscillations have also attracted a great deal of attention in the context of compact objects, neutron stars and black holes; consider, for example, the stability analysis of neutron stars using radial oscillation modes by Chandrasekhar [7]. More recently, interest in neutron star and black-hole oscillations has focused on their potential in the context of gravitational wave (GW) physics [8].

While most insight into stellar oscillations has been obtained from linear analysis (see, for example, [9-12]), nonlinear effects are known to play an important role in the phenomenology of oscillations in various scenarios. Examples include the nonlinear coupling of modes in the beat Cepheids (see, for example, [13]) and the saturation of $r$-mode oscillations and, thus, GW generation in rotating neutron stars first studied by Schenk et al. [14] and extended in [15-19]. More generally, perturbative studies have been extended to include nonlinear effects up to cubic order terms in the perturbations [20-22]. Mode coupling in neutron stars with particular regard to the generation of GWs has been investigated in the framework of higherorder perturbation theory in [23,24]; see also [25-28] for a gauge-invariant framework to compute higher-order perturbations including fluid backgrounds. While the present study focuses on neutron star oscillations, we emphasize that black-hole oscillations play an equally important role in GW physics $[29,30]$. At the linearized level, the corre- sponding solutions are given in the form of quasinormal modes [31-34] which dominate the signal from the late stages of a binary black-hole coalescence (see, for example, [31,35-37]). Nonlinearities in black-hole oscillations have been investigated in the framework of higherorder perturbation theory as well as numerical relativity [34,38]. A conclusive answer regarding the presence of nonlinear signatures in ring-down waveforms resulting from binary-black-hole inspiral and merger has as yet not been obtained, however, because of the high numerical accuracy required for such studies [31].

In comparison with black-hole configurations gravity is about an order of magnitude weaker in spacetimes containing neutron stars. This has motivated a variety of hydrodynamic simulations which implement gravitational effects in the form of some approximation such as Newtonian theory, the Cowling approximation or conformal flatness [39-44]. The present decade has seen dramatic progress in the numerical solution of the full Einstein field equations, however, and has resulted in fully relativistic simulations of single compact stars, collapse to black holes and neutron star binaries [45-53]. As in the case of blackhole simulations the available accuracy has not yet reached a level to facilitate high-precision studies of nonlinear effects, in particular, in the mildly nonlinear regime.

In consequence, there currently exists a gap in the literature studying in the fully nonlinear general relativistic framework with high-precision mildly and moderately nonlinear effects in oscillations of compact stars. The main purpose of the present paper is to fill this gap in the case of the simplest type of stellar pulsations, radial oscillations of spherically symmetric polytropic stellar models. We are aware that more realistic simulations of neutron stars require the inclusion of a multitude of microphysical effects such as more realistic equations of state and magnetic fields. For this reason, our study is intended in the first 
place to provide a general taxonomy of nonlinear features that will be encountered in the context of stellar pulsations. The results also provide valuable tools for calibrating the accuracy of general relativistic three-dimensional hydro codes.

Following Refs. [38,54], we achieve the necessary accuracy by formulating the problem in terms of deviations from an equilibrium background model, as is commonly done in traditional perturbation theory. In contrast to that approximation, however, we keep all terms of higher order in the deviations and thus arrive at a system of equations equivalent to the original nonlinear system. The main advantage of this approach is the elimination from the equations of all terms exclusively containing background quantities and, thus, the discretization error associated with these terms. The key improvement over the toy problem studied in [54] is the inclusion of the entire star including the important outer layers near the stellar surface.

This paper is organized in five sections. We set up the numerical framework in Sec. II. In Sec. III we study in detail the coupling of eigenmodes due to nonlinear effects, including a more detailed discussion of saturation and resonance effects. Section IV investigates two further nonlinear effects not directly concerning mode coupling, the stabilization of linearly unstable stars and the formation of discontinuities near the stellar surface. Finally, we summarize our findings in Sec. V. Throughout this work we adopt units corresponding to $c=G=1$, where $c$ is the speed of light and $G$ is the gravitational constant.

\section{NUMERICAL FRAMEWORK}

\section{A. Evolution equations}

The starting point for our description of a dynamic, spherically symmetric neutron star is the formulation developed by May and White $[55,56]$. Specifically, we write the metric in the form

$$
d s^{2}=-\lambda^{2} d t^{2}+\mu^{2} d x^{2}+r^{2}\left(d \theta^{2}+\sin ^{2} \theta d \phi^{2}\right),
$$

where $\lambda, \mu$ and areal radius $r$ are functions of time $t$ and radial position $x$. Unless stated otherwise, the coordinate $x$ is initialized by the areal radius of the background configuration (cf. Sec. II B below) and serves as a Lagrangian coordinate following the motion of the fluid elements during the time evolution. The neutron star is modeled as a single component perfect fluid at zero temperature. The corresponding energy momentum tensor can be expressed in the form $T^{\mu \nu}=(\rho+P) u^{\mu} u^{\nu}+P g^{\mu \nu}$, where $\rho$ and $P$ are energy density and pressure. The four velocity $u^{\mu}$ obeys the normalization condition $u^{\mu} u_{\mu}=-1$. Because the radial coordinate $x$ is comoving with the fluid elements we have the simple expression $u^{\mu}=\left[\lambda^{-1}, 0,0,0\right]$. We further assume rest mass conservation and neglect all heat transfer other than that due to the motion of the fluid. In particular, this excludes heat transfer by neutrinos or radiation as well as pair production and interaction with external fields. The equation of state (EOS) is modeled by a polytropic law $P=K \rho^{\gamma}$, where $K$ is the polytropic constant and $\gamma$ the polytropic index.

In order to achieve high accuracy near the boundaries, we find it important to employ variables with at most linear asymptotic behavior at both the center and the stellar surface. ${ }^{1}$ We therefore describe the stellar model in terms of a rescaled mass ${ }^{2}$ function $N$ and a function $\sigma$ which are defined by

$$
\begin{gathered}
N \equiv \frac{1}{2 r}\left(1-\frac{1}{\mu^{2}}\right), \\
\sigma=\frac{P}{\rho} .
\end{gathered}
$$

The set of variables is completed by the metric component $\lambda$ and an auxiliary velocity function $w \equiv r_{, t} / \lambda$ introduced to obtain a system of partial differential equations of first order in space and time.

The Einstein equations $G_{\mu \nu}=8 \pi T_{\mu \nu}$ and the conservation of energy and momentum $\nabla_{\mu} T^{\mu \nu}=0$ then result in the following equations:

$$
\begin{gathered}
\sigma_{, x}=-\frac{\lambda_{, x}}{\lambda}(1+\sigma)\left(1-\frac{\sigma}{C^{2}}\right), \\
N_{, x}=r_{, x}\left(4 \pi \rho-2 \frac{N}{r}\right), \\
N_{, t}=-w \lambda\left(4 \pi P+2 \frac{N}{r}\right), \\
r_{, t}=\lambda w, \\
w_{, t}=-\lambda(\rho+P)\left(\frac{w_{, x}}{r_{, x}}+\frac{2 w}{r}\right) .
\end{gathered}
$$

In these equations, commas are used to represent partial derivatives and $C^{2}=\partial p / \partial \rho$ is the sound speed. Only five of these equations are independent and we choose to use Eq. (9) in the determination of the eigenmodes below but not in the nonlinear time evolution.

In order to determine boundary conditions, we first consider the origin. Because the physical system is spherically symmetric by construction, all functions that are odd

\footnotetext{
${ }^{1}$ See Sec. V B.1 in [57] for a discussion of the difficulties arising from higher-order falloff of variables near the boundaries.

${ }^{2} \mathrm{~A}$ straightforward calculation shows that $m \equiv r^{2} N$ evaluated at the surface of the star is the mass parameter of the exterior vacuum metric in Schwarzschild coordinates.
} 
in the radius necessarily vanish at the origin, that is $r(0)=$ $0, N(0)=0$ and $w(0)=0$. The surface $x_{\mathrm{S}}$ of the star is defined as the radius where the pressure vanishes. We further define our time coordinate such that the interior solution matches to the Schwarzschild metric in the exterior. We thus obtain outer boundary conditions $P\left(x_{\mathrm{S}}\right)=0$, corresponding to $\sigma\left(x_{\mathrm{S}}\right)=0, \quad$ and $\quad \lambda\left(x_{\mathrm{S}}\right)=$ $\sqrt{1-2 N\left(x_{\mathrm{S}}\right) r\left(x_{\mathrm{S}}\right)}$.

\section{B. Deviations from an equilibrium configuration}

The purpose of this study is to analyze nonlinear effects in the time evolution of radial oscillations of a neutron star model with particular regard to the mildly nonlinear regime. Such an investigation requires numerical simulations of high accuracy. We achieve this by formulating the numerical evolution in terms of deviations from a stationary background configuration. In such a formulation all terms involving exclusively background quantities and, thus, the discretization errors associated with these terms drop out of the equations. Because we are mostly interested in scenarios where the deviations are small in comparison with the background terms, the elimination of these error terms leads to a significant increase in numerical accuracy.

This decomposition requires us to choose a convenient background. For the study at hand, the obvious choice is a spherically symmetric, static neutron star, as described by Tolman, Oppenheimer and Volkoff (TOV) [58,59]. The system of equations governing the TOV equilibrium configuration is given by the time independent limit of the system of Eqs. (4)-(8),

$$
\begin{gathered}
\bar{\sigma}_{, x}=-\frac{\bar{\lambda}_{, x}}{\bar{\lambda}}(1+\bar{\sigma})\left(1-\frac{\bar{\sigma}}{\bar{C}^{2}}\right) \\
\bar{N}_{, x}=\bar{r}_{, x}\left(4 \pi \bar{\rho}-2 \frac{\bar{N}}{\bar{r}}\right) \\
\frac{\bar{\lambda}_{, x}}{\bar{r}_{, x}}=\frac{\bar{\lambda}}{1-2 \bar{N} \bar{r}}(\bar{N}+4 \pi \bar{P} \bar{r}),
\end{gathered}
$$

where we have introduced an overbar to distinguish background variables from their time dependent counterparts. Note that $\bar{r}_{, x}=1$ if we initialize $x$ by the areal radius of the background model. Next, we formally introduce deviations from this equilibrium by

$$
f(x, t)=\bar{f}(x)+\Delta f(x, t) .
$$

Here and in the following, the function $f$ stands for either of the variables $\{\lambda, N, \sigma, \rho, P, r\}$. Following customary notation in the literature, we denote the radial displacement by $\xi \equiv \Delta r$.

We can now insert Eq. (13) into the set of evolution Eqs. (4)-(8). Of particular significance in the resulting expressions are the terms containing exclusively background quantities and no deviations. A straightforward calculation demonstrates that all these terms drop out of the equations because, by construction, the background variables $\bar{f}$ obey the stationary limit of Eqs. (4)-(8), that is, the TOV equations. We are left exclusively with terms of linear or higher order in the deviations $\Delta f$ and the evolution equations are given by

$$
\begin{aligned}
& \Delta \sigma_{, x}=-\frac{\bar{\sigma}_{, x}\left(\Delta \lambda C^{2}+\bar{\lambda} \Delta C^{2}\right)}{\lambda C^{2}}-\frac{\Delta \lambda_{, x}(1+\sigma)\left(C^{2}-\sigma\right)}{\lambda C^{2}} \\
& -\frac{\bar{\lambda}_{, x}\left[\Delta \sigma\left(C^{2}-\sigma\right)+(1+\bar{\sigma})\left(\Delta C^{2}-\Delta \sigma\right)\right]}{\lambda C^{2}} \\
& \Delta N_{, x}=\bar{r}_{, x} 4 \pi \Delta \rho+\xi_{, x} 4 \pi \rho \\
& -\frac{\bar{N}_{, x} \xi+\xi_{x} 2 N+\bar{r}_{, x}(2 \Delta N-4 \pi \bar{\rho} \xi)}{r} \\
& \Delta N_{, t}=-w \frac{\lambda}{r}(4 \pi r P+2 N) \\
& \xi_{, t}=\lambda w \\
& w_{, t}=(1-2 \bar{N} \bar{r}) \frac{\Delta \lambda_{, x}}{r_{, x}}+\left(w^{2}-2 \Delta N r-2 \bar{N} \xi\right) \frac{\lambda_{, x}}{r_{, x}} \\
& -\frac{\xi_{x}}{r_{, x}}(N \lambda+4 \pi \operatorname{Pr} \lambda)-\frac{\bar{r}_{, x}}{r_{, x}}(\Delta N \lambda-\Delta \lambda \bar{N}) \\
& -\frac{\bar{r}_{, x}}{r_{, x}} 4 \pi[\bar{P}(\bar{r} \Delta \lambda+\xi \lambda)+\Delta \operatorname{Pr} \lambda] .
\end{aligned}
$$

The boundary conditions for the deviations follow directly from those obtained for the full variables $f$ in Sec. II A. They are given by $\xi(0)=0, \Delta N(0)=0, w(0)=$ 0 as well as $\Delta \sigma\left(x_{\mathrm{s}}\right)=0$ and $\Delta \lambda(\lambda+\Delta \lambda)=-2(\xi N+$ $\bar{r} \Delta N)$.

\section{Eigenmodes}

In the limit of infinitesimally small deviations $\Delta f$ from the equilibrium solution $\bar{f}$, the time evolution of the star is governed by the linearized version of the system (14)-(18). The solutions to the linearized perturbation equations are given by an infinite set of oscillation modes with characteristic frequencies. This set of modes is a vital asset when interpreting the nonlinear time evolution. For each neutron star model, we therefore calculate the first 10 eigenmode profiles and their associated frequencies.

The linearized evolution system is most conveniently written in terms of the rescaled radial displacement $\zeta=$ $\bar{r}^{2} \xi / \bar{\lambda}$ (cf. chapter 26 in [60]) and reduces to the single partial differential equation

$$
W \zeta_{, t, t}=\frac{1}{\bar{r}_{, x}}\left(\frac{\Pi}{\bar{r}_{, x}} \zeta_{, x}\right)_{, x}+Q \zeta .
$$

Here, the variables $W, \Pi$ and $Q$ depend only on the 
background quantities. Exact expressions for these auxiliary functions are given in Eqs. (A1)-(A3).

Separation of variables straightforwardly implies a harmonic time dependence, i.e. $\zeta \sim e^{i \omega t}$, where $\omega$ is a frequency yet to be determined. The resulting equation represents a singular Sturm-Liouville problem, and the solutions $\zeta_{i}(x)$ of this eigenvalue problem form a complete orthonormal set. It is possible, therefore, to expand the radial displacement function $\zeta(t, x)$ in a series

$$
\zeta(t, x)=\sum_{i} A_{i}(t) \zeta_{i}(x)
$$

where the eigenmode coefficients $A_{i}(t)$ are given by the inner product

$$
A_{i}(t)=\left\langle\zeta, \zeta_{i}\right\rangle \equiv \int_{0}^{x_{\mathrm{S}}} W \zeta(t, x) \zeta_{i}(x) d x .
$$

The $A_{i}(t)$ thus represent a measure of how strongly an eigenmode $i$ contributes at a given time $t$.

\section{Numerical methods}

The equations for the TOV background (10)-(12) as well as the Sturm-Liouville problem (19) represent twopoint boundary-value problems which can be solved by means of a relaxation method [61]. We discretize the evolution system (14)-(18) using the implicit, secondorder accurate Crank-Nicholson scheme. From a numerical point of view, this implicit evolution scheme turns out to be conceptually identical to the two-point boundary-value problems of the background and eigenmode calculation. We therefore use the same relaxation algorithm for all of these calculations.

Finally, we compute the integrals appearing in the calculation of the inner products using a standard fourth-order accurate Simpson's Rule algorithm.

\section{E. Code tests}

The code has been tested in several ways. First, we have performed a convergence analysis for a polytropic model with $\gamma=2$ and $K=150 \mathrm{~km}^{2}$. We use 801, 1601 and 3201 grid points and obtain second-order convergence for the background model, the calculation of the eigenmode profiles and the time evolution including the eigenmode coefficients. In Fig. 1, we show the convergence factors of the $\ell_{2}$ norms of the evolution variables $\Delta \sigma, \Delta N$ and $\xi$ for a simulation of a perturbation given initially in the form of the first eigenmode with an amplitude of $10 \mathrm{~m}$. The convergence factor is defined as

$$
q \equiv \frac{\ell_{2}\left[f_{\mathrm{c}}\right]-\ell_{2}\left[f_{\mathrm{m}}\right]}{\ell_{2}\left[f_{\mathrm{m}}\right]-\ell_{2}\left[f_{\mathrm{f}}\right]},
$$

where the subscripts $c, m$ and $f$ stand for coarse, medium and fine resolution. For a second-order scheme and the above-mentioned grid setups we expect $q=4$. The minor

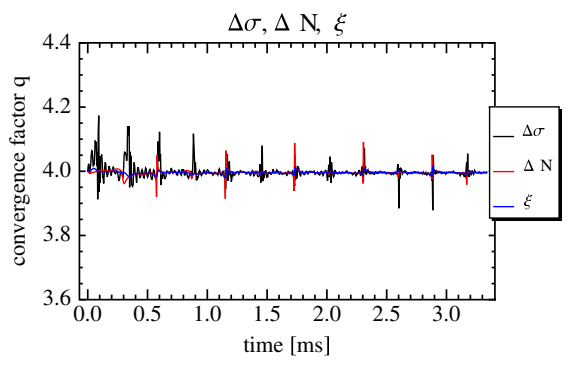

FIG. 1 (color online). The convergence factor $q$ of $\Delta \sigma, \Delta N$ and $\xi$ as functions of time. The obtained results are consistent with second-order convergence corresponding to $q=4$.

deviations from this value are due to zero crossings of the variables involved.

For the second test, we use the constraint Eq. (9) which vanishes in the continuum limit. In order to quantify the constraint violation due to the discretization error, we normalize the numerical constraint by the sum of the $\ell_{2}$ norms of the individual terms. We find the resulting normalized constraint violations to be of the order of $10^{-6}$ or less.

Third, we test the eigenmode calculation by checking the completeness of the eigenmode spectrum. This is done by calculating the weighted $\operatorname{sum} \sum_{i} A_{i}^{2} /\langle\zeta, \zeta\rangle$, which has to be unity due to the completeness of the basis. In our simulations we include the first 10 terms in this infinite series and obtain a maximal deviation from the expected value of 1 by about $10^{-4}$. As a by-product, this result demonstrates that the first 10 eigenmodes capture most of the dynamics of the system.

Finally, we compare the eigenmode frequencies with results available in the literature and observe excellent agreement; the maximal deviation in the first three eigenmodes is about $1 \%$ from results reported in [11] and less than $1 \%$ from those of [62].

Using the setup described in this section, we obtain highly accurate time evolutions. In fact, we observe that the overall error is dominated by the calculation of the inner products in Eq. (21) via the Simpson algorithm, rather than the time evolution of the grid variables. We believe this to be an artifact of the uncertainties in the eigenmode profiles themselves, the relative error being of the order of $10^{-5}$. Motivated by this assumption, we managed to further improve the accuracy using the fact that our time evolutions are typically dominated by one particular eigenmode. First, we identify this mode from the initial data construction. In the course of the evolution, we calculate the eigenmode coefficient associated with this mode. Before calculating the other eigenmode coefficients, however, we subtract the contribution of the dominating mode from the grid variables. By virtue of the orthogonality of the eigenmodes, this subtraction does not affect the other coefficients in the continuum limit. For finite numerical resolutions, however, the eigenmodes are not perfectly 
orthogonal and we avoid contamination of the overlap integrals due to the dominant mode.

Taking into account all numerical effects, we arrive at the following estimates for the uncertainties. All simulations discussed in the next section start from initial data consisting of a single mode. This mode is found to dominate the ensuing evolution and we measure its amplitude with a relative error of $10^{-5}$. All other modes are absent in the initial data, but are excited due to nonlinear effects. The accuracy of their measurement depends on their amplitude. For those modes used in our analysis we obtain relative uncertainties ranging from $10^{-2}$ for weak excitation to $10^{-5}$ for strong excitation.

\section{MODE COUPLING}

Before analyzing in detail the coupling of eigenmodes in our simulations, we need to address a conceptual difficulty arising from the nonlinear nature of hydrodynamics. Eigenmodes are, by construction, a feature of a linear theory and our calculation of the eigenmode spectrum in Sec. II C required us to specify a background configuration. Given a dynamic system evolved with the fully nonlinear theory, we analyze the deviations from that background configuration by projecting them onto the eigenmodes associated with that same background. The problem is that there exists no unique way of decomposing such a dynamic system into a background plus perturbations. Different background configurations imply different eigenmode spectra. In consequence of this ambiguity, we can interprete nonzero mode coefficients for modes not present in the initial data in two ways: (i) excitation due to nonlinear effects and (ii) a change in the background configuration and its eigenmode spectrum due to the finite amplitude of the initial perturbation. Both interpretations reflect the nonlinear nature of the theory and the nonuniqueness of choosing a background makes it impossible to distinguish in a well-defined manner between those interpretations. In the remainder of this work we will use the terminology mode coupling and excitation but, as a reminder of the ambiguities in the interpretation, we will put the words in italics. Note, however, that once a particular choice of the background has been specified, the ensuing projection of deviations from that background onto the corresponding eigenmode spectrum is uniquely defined. In that sense, the following analysis is selfconsistent and any study agreeing upon the same decomposition can be compared straightforwardly with our results.

Specifically, our analysis is based on the following construction of a background model plus nonlinear deviations. We consider either of the two stellar models labeled 1 and 2 in Table I. Numerically, we prescribe the initial perturbation of this background model by displacing the fluid elements from their equilibrium positions according to the profile of the displacement $\xi$ corresponding to one
TABLE I. Physical parameters for the three stellar models studied in this work.

\begin{tabular}{lccccc}
\hline \hline Model & $\gamma$ & $K$ & $\rho_{\mathrm{c}}\left[\mathrm{g} / \mathrm{cm}^{3}\right]$ & $M\left[M_{\odot}\right]$ & $R[\mathrm{~km}]$ \\
\hline 1 & 2 & $150 \mathrm{~km}^{2}$ & $2.022 \times 10^{15}$ & 1.555 & 10.82 \\
2 & 2.25 & $700 \mathrm{~km}^{2.5}$ & $3.600 \times 10^{15}$ & 1.584 & 8.414 \\
3 & 2 & $150 \mathrm{~km}^{2}$ & $3.774 \times 10^{15}$ & 1.655 & 9.222 \\
\hline \hline
\end{tabular}

single eigenmode. From this displacement, we straightforwardly calculate the profiles for the remaining evolution variables $\Delta \lambda, \Delta \sigma$ and $\Delta N$ according to Eqs. (18), (14), and (15) in that order. Finally, we set the radial velocity $w$ to zero. In the remainder of this work, we always label the single mode present in the initial data as $j$ and denote its amplitude in the form of its surface displacement $\xi_{j} \equiv$ $\xi\left(x_{\mathrm{S}}\right)$ Similarly, we use the label $i$ to identify all modes present in the time evolution.

During the evolution, we measure the presence of a given mode $i$ in the form of the associated eigenmode coefficient $A_{i}(t)$. In practice, we find these coefficients to oscillate periodically; cf. Figures 2 and 3. It is convenient, therefore, to use the resulting oscillation maximum $A_{i}=$ $\max \left|A_{i}(t)\right|$ as an overall measure of the degree of excitation of that mode in the entire evolution. The intuitive interpretation of the resulting maxima is greatly helped by their one-to-one relation with the corresponding displacement of the stellar surface. We recall for this purpose Eq. (20). By setting $A_{k}=1$ and $A_{i}=0$ for all $i \neq k$ in that relation, we can evaluate the surface displacement $\zeta\left(x_{\mathrm{S}}\right)$ and, thus, $\xi\left(x_{\mathrm{S}}\right)$ corresponding to eigenmode $k$ with unit amplitude $A_{k}$. In Table II, we list the resulting surface displacements for both stellar models studied in this section. It becomes clear from the table that we expect to deal with numerically small values of the coefficients $A_{k} \ll 1$.

In the remainder of this section we will analyze in detail how initial data given in the form of one single eigenmode gives rise to the excitation of other modes.

\section{A. Model 1: A $\gamma=\mathbf{2}$ polytrope \\ 1. Exciting the fundamental mode}

We first focus on the coupling of eigenmodes in the case of model 1, a $\gamma=2$ polytrope which is stable against (linear) radial perturbations. Our first analysis is based on an initial displacement of the fluid elements given by the profile of the fundamental eigenmode $j=1$. The simulations last for a physical time of $5 \mathrm{~ms}$ and the amplitude of the initial data is varied between $10 \mathrm{~cm}$ and $70 \mathrm{~m}$. For illustration, we show in Fig. 2 the time evolution of the eigenmode coefficients $A_{1}, \ldots, A_{4}$ obtained for an initial surface displacement $\xi_{1}=10 \mathrm{~m}$. Higher-order modes show a similar behavior with decreasing amplitude. Note that the coefficients $A_{i}$ corresponding to weakly excited modes such as $i=3$ and $i=4$ in the figure do not oscillate around zero but reveal an offset. This effect is not surpris- 
A1

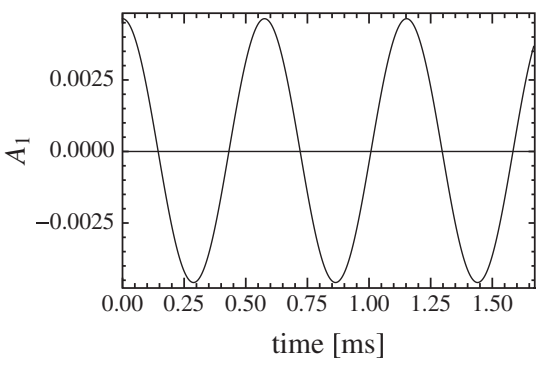

A3

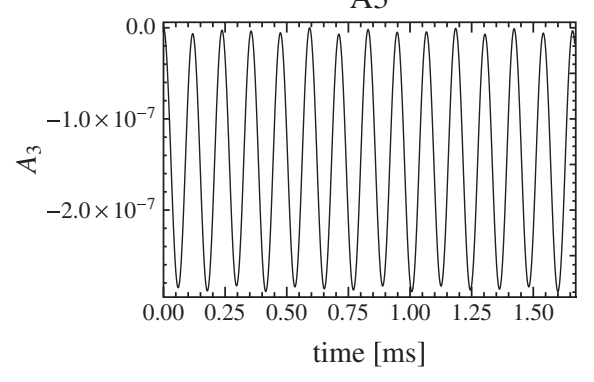

$\mathrm{A} 2$
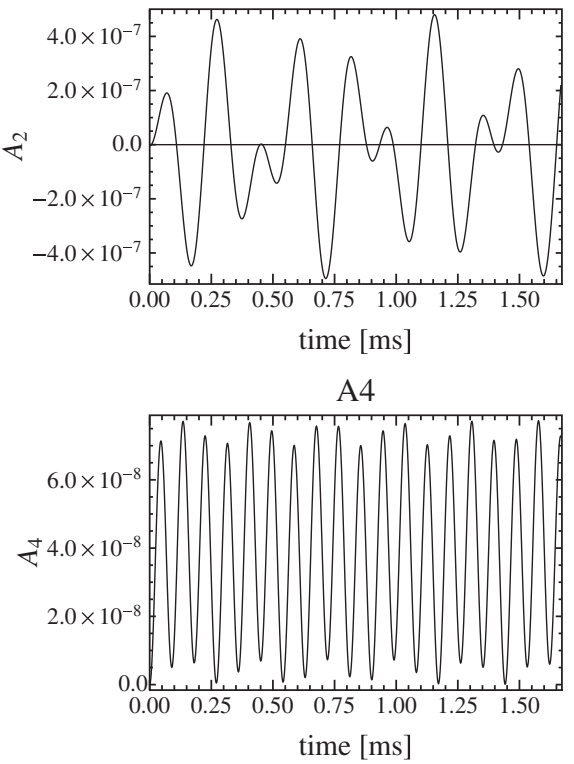

FIG. 2. The evolution of the first four eigenmode coefficients $A_{i}$ obtained for model 1 and an initial displacement corresponding to the fundamental mode with a surface amplitude of $\xi_{1}=10 \mathrm{~m}$.

ing bearing in mind that the sinusoidal dependence of the eigenmode oscillations is a direct consequence of the separation of variables in Eq. (19) according to $\zeta(t, x)=$ $\zeta_{i}(x) e^{i \omega_{i} t}$. In the linear limit we expect an eigenmode to describe a stellar oscillation around the equilibrium configuration. In the present case, however, the weak eigenmodes have to be viewed as perturbations of a time dependent background, namely, the equilibrium configuration plus any strongly excited eigenmodes. The observed offset thus arises as one of the nonlinear effects of the system. Indeed, we empirically find that it increases quadratically with the amplitude of the initial data and disappears as we reduce the initial displacement of the fluid elements to zero.

The excitation of higher-order modes inevitably corresponds to a flow of energy away from the initially present mode. Whereas this effect is too small in the example of

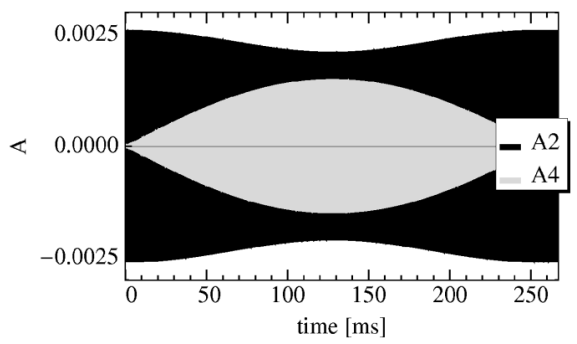

FIG. 3. The envelopes of the amplitudes of the second and fourth eigenmode coefficients $A_{i}$ obtained for model 2 and an initial displacement corresponding to the second mode with a surface amplitude of $\xi_{2}=10 \mathrm{~m}$. Note that the excitation of mode $i=4$ is so strong that it leads to a visible temporary decrease in $A_{2}(t)$.
Fig. 2 to be noticeable in the amplitude $A_{1}(t)$, the strong coupling between modes 2 and 4 in the case of model 2 in Fig. 3 demonstrates a strong amplitude modulation of the mode coefficients. We will discuss this exceptionally strong coupling in more detail in the context of resonance effects in Sec. III D below.

As mentioned above, we use the maxima for each of the $\left|A_{i}(t)\right|$ to determine the degree of excitation of the individual modes. The resulting values for the first six eigenmodes are shown in Fig. 4. We have ignored higher-order modes because they are very weakly excited and therefore subject to uncertainties larger than the limit discussed in Sec. II E.

The results in the figure show that the amplitude of the fundamental eigenmode grows linearly with the initial amplitude $\xi_{1}$, as expected. In contrast, all other eigenmode coefficients exhibit a quadratic dependence on $\xi_{1}$

$$
A_{i}=c_{i, 1}\left(\xi_{1}\right)^{2} .
$$

The expansion coefficients $c_{i, 1}$ obtained from regression are listed in Table III. Throughout the entire amplitude range we do not observe any signs of a transition of the power laws from quadratic to higher order. This indicates that the nonlinear interaction is dominated by leadingorder effects. This changes when we consider different initial data.

\section{Exciting higher modes}

Next, we consider initial perturbations in the form of the second and the third eigenmode, respectively. The corresponding plots, now up to and including mode10, are shown in Fig. 5. As before the eigenmode coefficient of the initially present mode shows linear behavior. For suffi- 
TABLE II. Surface displacement $\xi\left(x_{\mathrm{s}}\right)$ corresponding to eigenmode $k$ with unit amplitude $A_{k}=1$ for the first two stellar models of Table I.

\begin{tabular}{lccccc}
\hline \hline Mode $\xi\left(x_{\mathrm{S}}\right)[\mathrm{km}]$ model 1 & $\xi\left(x_{\mathrm{S}}\right)$ & {$[\mathrm{km}]$ model } & 2 & Mode $\xi\left(x_{\mathrm{S}}\right)$ & {$[\mathrm{km}]$ model $1 \xi\left(x_{\mathrm{S}}\right)[\mathrm{km}]$ model 2 } \\
\hline 1 & 2.15 & 1.54 & 6 & 28.64 & 14.45 \\
2 & 6.31 & 3.84 & 7 & 35.47 & 17.43 \\
3 & 11.08 & 6.29 & 8 & 42.74 & 20.51 \\
4 & 16.41 & 8.88 & 9 & 50.42 & 23.70 \\
5 & 22.27 & 11.60 & 10 & 58.49 & 26.98 \\
\hline \hline
\end{tabular}

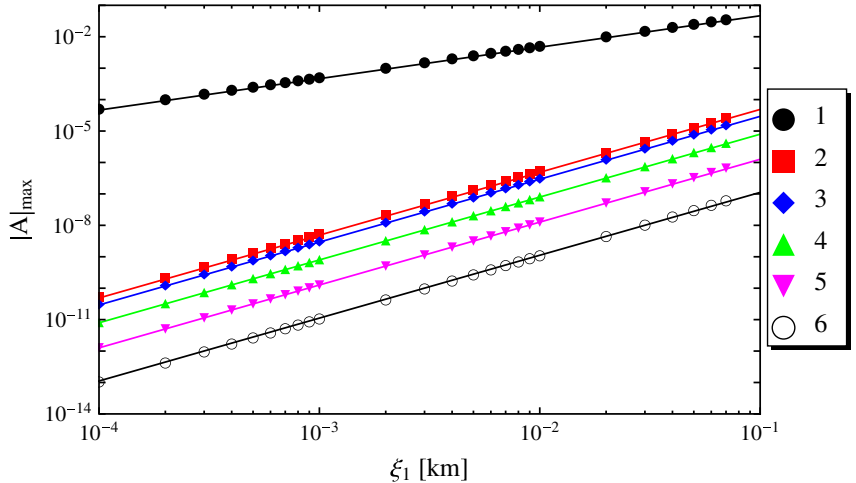

FIG. 4 (color online). The maximal eigenmode coefficients of the first six eigenmodes as functions of the amplitude of the initial perturbation in form of the fundamental mode. The curves represent a linear function in $\xi_{1}$ for the fundamental mode and quadratic power laws for all other modes.

TABLE III. The quadratic expansion coefficients for the mode coupling between the fundamental and other modes.

\begin{tabular}{|c|c|c|c|c|c|}
\hline$i$ & 2 & 3 & 4 & 5 & 6 \\
\hline \multicolumn{6}{|c|}{$c_{i, 1}\left[\mathrm{~km}^{-2}\right] 4.9 \times 10^{-3} 2.9 \times 10^{-3} 7.9 \times 10^{-4} 1.3 \times 10^{-4} 1.1 \times 10^{-5}$} \\
\hline
\end{tabular}

ciently small amplitudes of the initial displacement, we also observe the quadratic power law for all modes not present in the initial data. For larger perturbations, however, these show a clear transition to higher-order power

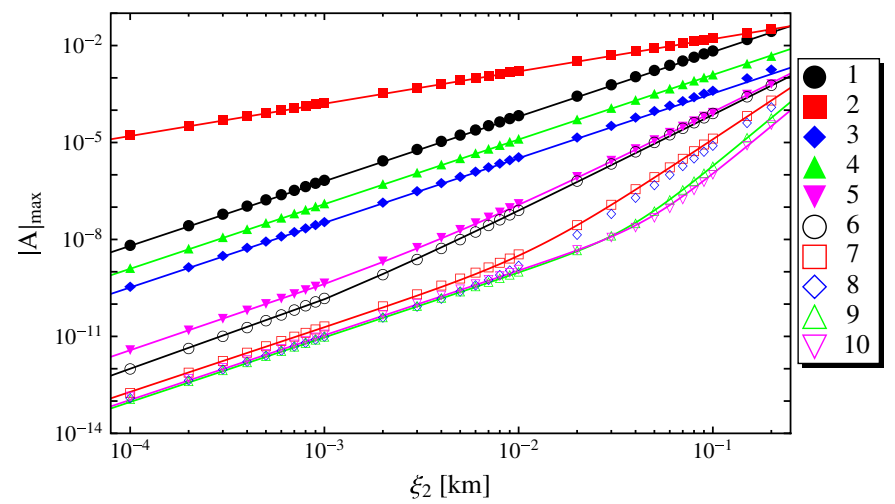

laws. This transition demonstrates a significant contribution of couplings beyond the leading-order terms and we will refer to this regime as the moderately nonlinear regime as opposed to the weakly nonlinear regime where quadratic coupling terms dominate. The details of the transition such as the threshold amplitude of the initial data and the relative significance of the higher-order terms depend on the individual mode under consideration as well as on the choice of initial data. We empirically investigate the transition by fitting the eigenmode coefficients according to

$$
A_{i}=c_{i, j}\left(\xi_{j}\right)^{2}+d_{i, j}\left(\xi_{j}\right)^{3}+e_{i, j}\left(\xi_{j}\right)^{4}+f_{i, j}\left(\xi_{j}\right)^{5}+\ldots
$$

In practice, we find this series to be dominated by a subset of the terms on the right-hand side and we explicitly set subdominant coefficients to zero. The resulting coefficients are listed in Table IV and the corresponding fits are shown as the lines in Figs. 4-6. While there appears to be a tendency for the expansion coefficients $c_{i, j}, d_{i, j}, \ldots$ to decrease for larger mode numbers $i$, there are exceptions to this rule. Consider, for example, $c_{3,2}$ and $c_{4,2}$ which demonstrate that the second mode couples more strongly to mode 4 than to mode 3 . This is corroborated by the data for mode 3 (filled diamonds) and 4 (filled upward triangle) in the left panel of Fig. 5. It is interesting to note in this context that the frequency ratio of modes 2 and 4 is close to $1: 2$. We will return to the issue of resonance phenomena

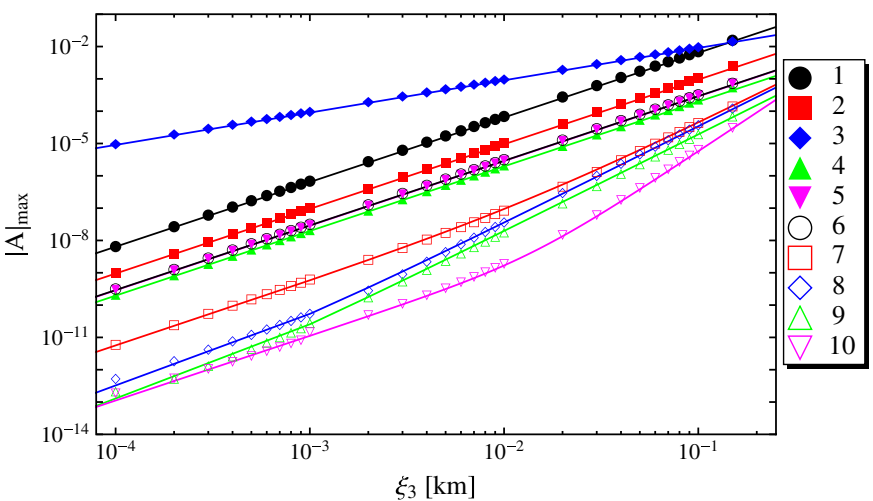

FIG. 5 (color online). The maximal eigenmode coefficients of the first ten eigenmodes as functions of the amplitude of the initial perturbation in form of the second (left panel) and the third (right panel) mode. 
TABLE IV. Expansion coefficients for model 1 obtained from fitting Eq. (24) to the data points for initial data in the form of mode $j=2$ and $j=3$, respectively.

\begin{tabular}{lccccccc}
\hline \hline$i$ & $c_{i, 2}\left[\mathrm{~km}^{-2}\right]$ & $d_{i, 2}\left[\mathrm{~km}^{-3}\right]$ & $e_{i, 2}\left[\mathrm{~km}^{-4}\right]$ & $f_{i, 2}\left[\mathrm{~km}^{-5}\right]$ & $c_{i, 3}\left[\mathrm{~km}^{-2}\right]$ & $d_{i, 3}\left[\mathrm{~km}^{-3}\right]$ & $e_{i, 3}\left[\mathrm{~km}^{-4}\right]$ \\
\hline 1 & 0.63 & 0 & 0 & 0 & 0.64 & 0 \\
2 & $\cdots$ & $\cdots$ & $\cdots$ & $\cdots$ & 0.095 & 0 \\
3 & 0.033 & 0 & 0 & 0 & $\cdots$ & 0 \\
4 & 0.12 & 0 & 0 & 0 & 0.020 & 0 \\
5 & $4.1 \times 10^{-4}$ & 0.078 & 0 & 0 & 0.029 & 0 & 0 \\
6 & $1.3 \times 10^{-4}$ & 0.069 & 0 & 0 & 0.029 & 0 \\
7 & $1.9 \times 10^{-5}$ & 0 & 0.118 & 0 & $5.8 \times 10^{-4}$ & 0.040 \\
8 & $9.4 \times 10^{-6}$ & 0 & 0.073 & 0 & $4.9 \times 10^{-5}$ & 0.034 \\
9 & $9.3 \times 10^{-6}$ & 0 & 0 & 0.172 & $2.3 \times 10^{-5}$ & 0 \\
10 & $1.1 \times 10^{-5}$ & 0 & $\ldots$ & 0.101 & $1.1 \times 10^{-5}$ & 0 \\
11 & $\cdots$ & $\cdots$ & $\cdots$ & $\cdots$ & $1.1 \times 10^{-5}$ & 0 \\
12 & $\cdots$ & $\cdots$ & $\cdots$ & $1.2 \times 10^{-5}$ & 0 \\
\hline \hline
\end{tabular}

in Sec. III D. Another feature visible both in the expansion coefficients in Table IV and the curves in Fig. 5 is that the modes excited due to nonlinear effects form groups of similar polynomial behavior. For $j=2$ they form pairs and for $j=3$ triplets. As a function of the initial amplitude of mode 2 (mode 3 ), for example, modes 5 and 6 (modes 7 , 8 and 9) show a transition to a cubic power law and modes 7 and 8 (modes 10, 11 and 12) a transition to a fourth-order power law. See also the block diagonal structure in Table IV listing the coefficients $d_{i, 2}, e_{i, 2}$ and $f_{i, 2}$ (coefficients $d_{i, 3}$ and $e_{i, 3}$ ).

To summarize our findings, we observe a weakly nonlinear regime in which the amplitude of secondary modes grows quadratically with the amplitude of the initial displacement. In the case of $j=2$ or $j=3$ and sufficiently large amplitudes, the mode excitation exhibits a transition to higher-order power laws. Secondarily excited modes form multiplets; for initial data in the form of mode $j>$ 1 , the excitation of modes $i=j+1, \ldots, 2 j$ depends quadratically on the initial amplitude, modes $i=2 j+1, \ldots, 3 j$ show a transition to a third-order power law, modes $i=$ $3 j+1, \ldots, 4 j$ a transition to a fourth-order power law and so on. Finally, higher-order modes appear to have a significantly stronger tendency to transfer energy to lowerorder modes than the other way around. The latter observation was also indicated by Papadopoulos and Sopuerta's [38] study of black-hole oscillations and suggested to explain the robustness of black holes to strong deformations.

\section{B. Model 2: A stiffer equation of state}

We now turn our attention to model 2 of Table I. We want to assess to what extent the observations in the previous section depend on the equation of state of the stellar model. As before, we consider three scenarios where we prescribe initial data in the form of mode $j=1,2$ or 3 and measure the degree of excitation of the other modes up to and including $i=10$ due to nonlinear effects. It is reassuring to see that using this model we qualitatively reproduce most of the general features discussed above. For comparison with the results for model 1, we show in Tables V and VI the expansion coefficients and in Fig. 6 the eigenmode coefficients resulting from initial data in the form of eigenmode $j=2$ or 3 .

While confirming the overall behavior observed for model 1, however, the analysis of model 2 reveals additional complications arising from two effects which were barely visible in the analysis of model 1: saturation and resonance.

\section{Saturation}

We first discuss the saturation effect observed in the analysis of model 2. Consider for this purpose the left panel of Fig. 6 which shows the excitation of modes resulting for $j=2$. We first notice a relatively strong excitation of mode 4; compare the filled upper triangles in this plot with their counterparts in Fig. 5 for model 1. Indeed, the excitation of mode 4 is so strong that the resulting power law fit crosses the curve for mode 2 at initial amplitudes just above $10^{-2} \mathrm{~km}$. Note, however, how the actual mode coefficients for mode 4 (filled upper triangles) start deviating from the polynomial fit at such large amplitudes; the actual excitation of mode 4 is weaker than expected from extrapolation of the polynomial fit and the coefficients $\left|A_{4}\right|_{\max }$ remain below those of the initially present mode $\left|A_{2}\right|_{\max }$. It appears that at sufficiently large amplitudes, mode 4 starts acting as a significant source of excitation itself and transfers energy to other modes via

TABLE V. The quadratic expansion coefficients $c_{i, 1}$ for the mode coupling between the fundamental and other modes for the stiffer neutron star model.

\begin{tabular}{lccccc}
\hline \hline$i$ & 2 & 3 & 4 & 5 & 6 \\
\hline$c_{i, 1}\left[\mathrm{~km}^{-2}\right]$ & 0.032 & 0.011 & $1.5 \times 10^{-3}$ & $7.6 \times 10^{-5}$ & $8.9 \times 10^{-6}$ \\
\hline \hline
\end{tabular}



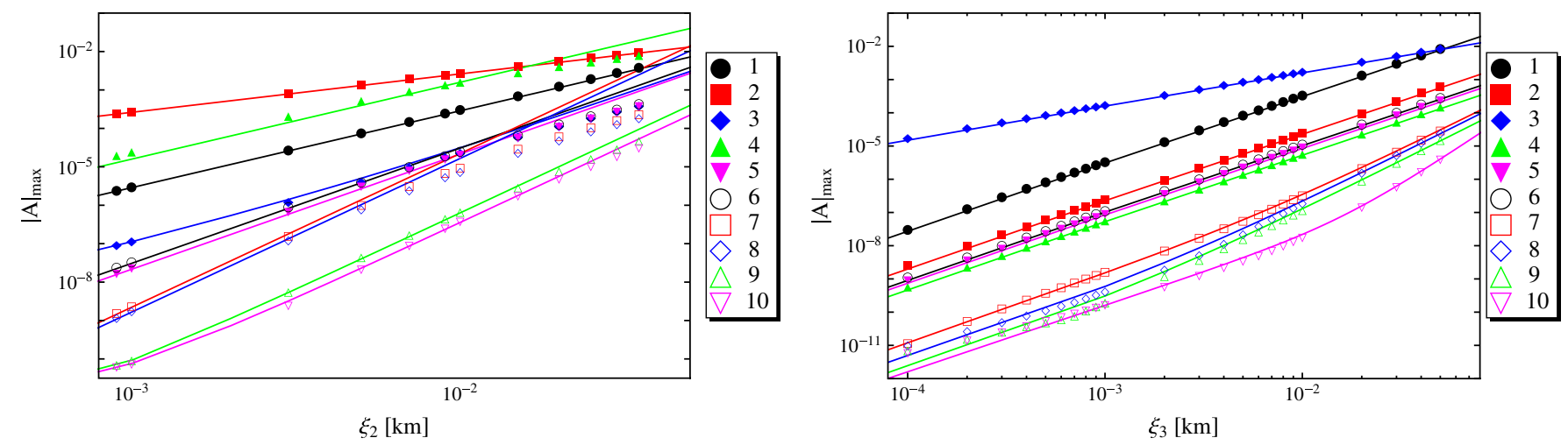

FIG. 6 (color online). The maximal eigenmode coefficients of the first ten eigenmodes as functions of the amplitude of the initial perturbation in form of the second eigenmode (left panel) or the third eigenmode (right panel) as obtained for model 2.

nonlinear coupling. The equilibrium reached in this way sets a limit for the growth in amplitude of mode 4. On closer investigation of the left panel of Fig. 6, we notice a similar behavior for modes 5 to 8 . These modes show saturation at smaller amplitudes which is compatible with our general observation that higher-order modes tend to transfer energy to lower-order modes more efficiently than the other way round.

We conclude from this observation that for the present example the assumption of two-mode coupling would oversimplify the situation. An accurate modeling of the chain of excitation whereby energy is transferred from mode 2 via mode 4 to other modes requires us to take into account higher-order effects in the mode coupling as provided, for example, by our fully nonlinear numerical treatment.

\section{Resonance effects}

Resonance is a well-known phenomenon arising in the context of the forced oscillator. Following Van Hoolst [22], we can model the stellar oscillations as a system of forced oscillators. The time evolution of the oscillation amplitudes is then determined by

$$
\frac{d^{2} A_{i}}{d t^{2}}+\omega_{i}^{2} A_{i}=\frac{a_{0}}{2}+\sum_{n}\left(a_{n} \sin n \Omega t+b_{n} \cos n \Omega t\right)
$$

Here $\Omega$ is the frequency and the $a_{n}$ and $b_{n}$ are the amplitudes of the driving forces. Assuming for simplicity that the $b_{n}$ vanish, Eq. (25) has the analytic solution

$$
A_{i}(t)=\sum_{n} \frac{a_{n}}{\omega_{i}^{2}-(n \Omega)^{2}} \sin n \Omega t \quad n \epsilon \mathcal{N} .
$$

In our case, the driving terms are typically dominated by the single eigenmode $j$ present in the initial data and therefore $\Omega=\omega_{j}$. This mode should excite with particular efficiency those modes which have an eigen frequency close to an integer multiple of $\omega_{j}$. In Table VII we show the frequencies and corresponding ratios for some modes of model 2 whose frequencies have ratios close to integer values. Resonance occurs most conspicuously between modes 2 and 4 and we have already noted the strong excitation of mode 4 in the left panel of Fig. 6. The effect is not as dramatic in the case of modes 6 and 8, but we still observe a preferred excitation of these modes compared with model 1; compare, for example, their excitation with that of mode 3 and the corresponding results in the left panel of Fig. 5. We also note a small deviation in the

TABLE VI. Expansion coefficients for model 2 obtained from fitting Eq. (24) to the data points for initial data in the form of mode $j=2$ and 3 , respectively.

\begin{tabular}{lccccccc}
\hline \hline$i$ & $c_{i, 2}\left[\mathrm{~km}^{-2}\right]$ & $d_{i, 2}\left[\mathrm{~km}^{-3}\right]$ & $e_{i, 2}\left[\mathrm{~km}^{-4}\right]$ & $f_{i, 2}\left[\mathrm{~km}^{-5}\right]$ & $c_{i, 3}\left[\mathrm{~km}^{-2}\right]$ & $d_{i, 3}\left[\mathrm{~km}^{-3}\right]$ & $e_{i, 3}\left[\mathrm{~km}^{-4}\right]$ \\
\hline 1 & 2.8 & 0 & 0 & 0 & 3.14 & 0 & 0 \\
2 & $\cdots$ & $\cdots$ & $\cdots$ & $\cdots$ & 0.22 & 0 & 0 \\
3 & 0.09 & 0 & 0 & 0 & $\cdots$ & $\cdots$ & 0 \\
4 & 24 & 0 & 0 & 0 & 0.053 & 0 & 0 \\
5 & $2.1 \times 10^{-3}$ & 21 & 0 & 0 & 0.084 & 0 & 0 \\
6 & $5.0 \times 10^{-4}$ & 31 & 0 & 0 & 0.099 & 0 & 0 \\
7 & $2 \times 10^{-6}$ & 0 & 2210 & 0 & $1.3 \times 10^{-3}$ & 0.22 & 0.18 \\
8 & $<2 \times 10^{-6}$ & 0 & 1650 & 0 & $4.1 \times 10^{-4}$ & 0.11 & 0 \\
9 & $<2 \times 10^{-6}$ & 0 & 62.8 & 0 & $2.0 \times 10^{-4}$ & 0 & 0.52 \\
10 & $<2 \times 10^{-6}$ & 0 & 35.2 & 0 & $1.5 \times 10^{-4}$ & 0 & \\
\hline \hline
\end{tabular}


TABLE VII. The frequencies and the most promising resonance factors $\omega_{2 i} / \omega_{i}$ or $\omega_{3 i} / \omega_{i}$ for some of the eigenmodes of model 1 and model 2 .

\begin{tabular}{lcccccc}
\hline \hline & \multirow{2}{*}{ Model 1 } & Model 2 & \multicolumn{2}{c}{ Model 1 } & \multicolumn{2}{c}{ Model 2 } \\
Mode $i$ & $\omega_{i}[\mathrm{kHz}]$ & $\omega_{i}[\mathrm{kHz}]$ & $\omega_{2 i} / \omega_{i}$ & $\omega_{3 i} / \omega_{i}$ & $\omega_{2 i} / \omega_{2}$ & $\omega_{3 i} / \omega_{i}$ \\
\hline 1 & 10.908 & 11.197 & $\ldots$ & $\ldots$ & $\ldots$ & $\ldots$ \\
2 & 35.299 & 47.526 & 1.975 & 2.890 & 2.000 & 2.939 \\
3 & 53.011 & 72.024 & 1.925 & $\ldots$ & 1.940 & $\ldots$ \\
4 & 69.711 & 95.065 & 1.919 & $\ldots$ & 1.931 & $\ldots$ \\
5 & 85.985 & 117.527 & $\ldots$ & $\ldots$ & $\ldots$ & $\ldots$ \\
6 & 102.026 & 139.694 & $\ldots$ & $\ldots$ & $\ldots$ & $\ldots$ \\
7 & 117.935 & 161.685 & $\ldots$ & $\ldots$ & $\ldots$ & $\ldots$ \\
8 & 133.756 & 183.570 & $\ldots$ & $\ldots$ & $\ldots$ & $\ldots$ \\
\hline \hline
\end{tabular}

transition to different power laws in the moderately nonlinear regime. Consider modes $i=9,10$ in the case of $j=$ 2 in Table VI. According to the rule, the excitation of these modes should exhibit a transition to fifth order in $\xi_{2}$, but instead we observe a fourth-order dependence. We believe this to be a consequence of the strong excitation of mode 4, so that our approximation of a single strong mode driving the coupling is no longer valid.

In all cases of mode coupling, we observe a periodic transfer of energy back and forth between the eigenmodes involved. This manifests itself in a modulation of the oscillation amplitude as apparent, for example, in Fig. 2, in particular, the upper right panel. Such amplitude modulation is also present in the case of strong resonance. In contrast to "normal" coupling of eigenmodes, however, we further observe in cases of strong resonance a modulation period which depends sensitively on the amplitude of the initial data. We illustrate this in Fig. 7 which displays the coefficient $A_{4}(t)$ obtained for model 2 and initial data in the form of the second eigenmode with different amplitudes. As is evident in the figure, larger amplitudes result in shorter modulation periods. The resulting numerical values of the modulation frequency are shown in Table VIII and are well approximated by the linear relation

$$
\Omega_{\text {mod }}\left(\xi_{2}\right)=7.55 \mathrm{~Hz}+577 \mathrm{~Hz} \frac{\xi_{2}}{\mathrm{~km}} .
$$

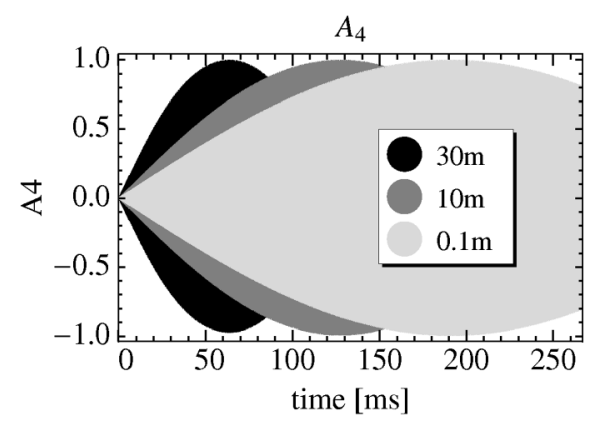

FIG. 7. The evolution of the fourth eigenmode coefficient for different perturbation amplitudes.
TABLE VIII. This table presents the frequencies obtained by fitting the envelopes of the eigenmode coefficients evolution with sinusoidal functions.

\begin{tabular}{lc}
\hline \hline Initial amplitude $[\mathrm{m}]$ & Frequency $\Omega[\mathrm{Hz}]$ \\
\hline 0.1 & 8.21 \\
1 & 8.27 \\
10 & 12.29 \\
20 & 18.98 \\
30 & 25.30 \\
\hline \hline
\end{tabular}

In the perturbative limit $\xi_{2} \rightarrow 0$ we obtain a modulation frequency of $\Omega_{\text {mod }}=7.55 \mathrm{~Hz}$. A detailed interpretation in the context of coupled harmonic oscillators [22] is beyond the scope of this paper, largely because it is highly nontrivial to calculate the coupling constants. We note, however, the close resemblance between the measured modulation frequency and the difference between the involved eigenmodes' frequencies

$$
\Omega_{\mathrm{mod}} \approx \frac{\delta \omega}{2}=\frac{\omega_{4}-2 \cdot \omega_{2}}{2} \approx 6.5 \mathrm{~Hz}
$$

Note that $\delta \omega$ is orders of magnitude below the individual frequencies $\omega_{2}$ and $\omega_{4}$ and therefore subject to a larger relative error. Within this error, the measured value agrees with the prediction of Eq. (27).

Leaving a detailed investigation for future work, we tentatively interpret our observations as follows. The modulation frequency is closely related to the difference in the frequencies of the coupling modes and decreases significantly as we approach resonance. As has already been discussed in Sec. III C, nonlinear effects set a limit on the resonance. They further result in a deviation of the modulation frequency from the limit of perfect resonance $\Omega_{\text {mod }} \rightarrow 0 \mathrm{~Hz}$.

In comparison, the resonance between modes 2 and 4 of model 1 is less pronounced and we find the modulation frequency to be independent of the amplitude of the initial data. Indeed, Eq. (28) predicts $\delta \omega=141 \mathrm{~Hz}$ for model 1 . This value is an order of magnitude larger than that obtained for model 2 and appears to be much more robust to effects of a finite initial amplitude $\xi_{2}$ as given by the second term on the right-hand side of Eq. (27).

\section{FURTHER NONLINEAR EFFECTS}

In this section we consider two nonlinear effects which are not directly related to the coupling of eigenmodes. First, we study the stability properties of a stellar model close to the maximum of the mass-radius relation but located on the unstable branch. From linear theory, we would expect this model to be unstable to small perturbations away from its equilibrium configuration. Second, we investigate the consequences of the vanishing of the speed 
of sound at the stellar surface with regard to the formation of discontinuities near the surface.

\section{A. Stabilization of linearly unstable stars}

A particularly interesting scenario for the investigation of nonlinear effects is that of marginally stable neutron stars. It is well known that neutron stars of sufficient compactness become unstable with respect to their fundamental radial oscillation mode [7]. The onset of this instability occurs at zero frequency of the fundamental mode. At this point linear perturbation terms cancel in the equations and the higher-order perturbations become more important.

We study this effect quantitatively for model 3 of Table I. We have already mentioned that this model is unstable, i.e. its fundamental oscillation mode has an imaginary frequency $\omega_{1}^{2}=-779 \mathrm{~Hz}^{2}$. We test the prediction of the linearized theory by prescribing initial data in the form of this fundamental eigenmode using an amplitude of $10 \mathrm{~m}$. The surprising result of the fully nonlinear time evolution is shown in Fig. 8; the star oscillates periodically over many milliseconds without any sign of instability. We find this general behavior independent of whether the initial displacement represents an expansion (dashed curve) or a compression (solid curve) of the star, even though these two scenarios differ in the details of the ensuing oscillations. This result demonstrates that nonlinear effects have the capacity to stabilize a linearly unstable neutron star. It will be interesting in future work to study this effect in more detail from an analytic point of view.

In addition to this qualitative difference between perturbative predictions and the nonlinear evolution, we observe a variety of quantitative deviations. Contrary to expectations from linearized theory, the sign of the initial perturbation of the star has an impact on the resulting oscillation pattern. In particular, the frequency and amplitude of the oscillation differ significantly for the two cases as is apparent in Fig. 8. We further observe an offset of the eigenmode coefficient indicating that the star is no longer oscillating symmetrically around its equilibrium position. We have already identified such an offset as a nonlinear effect in Sec. III A 1. In the present example, however, the offset is much larger. We further note that the solid curve

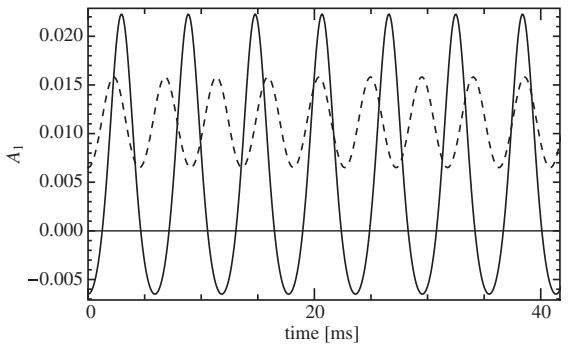

FIG. 8. The coefficient of the fundamental eigenmode. The dashed line corresponds to an initial expansion of the star, the solid line to a compression.

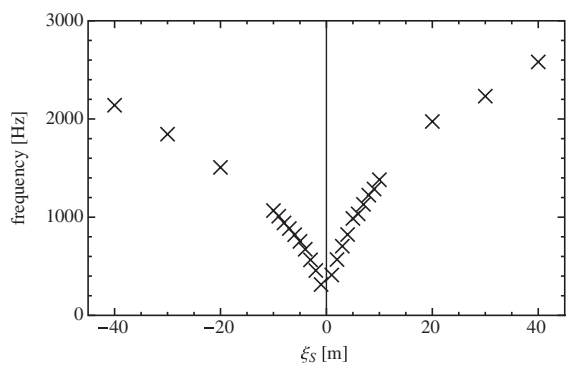

FIG. 9. The frequency of the fundamental mode as function of the initial perturbation. The frequencies have been obtained by a Fourier analysis of the nonlinear evolution.

does not have sinusoidal shape but is visibly distorted. Finally, we find that the oscillation frequency of the nonlinear evolution depends not only on the sign but also on the initial amplitude of the perturbation. Indeed, the measured values differ substantially from those predicted by linear theory. This is illustrated in Fig. 9, where we show the oscillation frequency as a function of the amplitude in the range $-40 \mathrm{~m}$ to $+40 \mathrm{~m}$. As expected intuitively, we obtain larger real frequencies, i.e. larger deviations from the linear prediction, for larger amplitudes of the initial data. Conversely, we recover the linear limit and observe collapse of the stellar model when choosing initial perturbations of sufficiently small amplitude, in the decimeter range for the present example.

\section{B. Shock formation at the surface}

Analysis of the asymptotic structure of the TOV model combined with a polytropic equation of state (cf. Sec. 5.2.5 of [62]) shows that the speed of sound vanishes as $\left(\bar{r}_{\mathrm{S}}-\right.$ $\bar{r})^{1 / 2}$ at the surface regardless of the parameters of the polytrope. Here $\bar{r}_{\mathrm{S}}$ is the radius of the star. Because of this decrease of the speed of sound, a signal of finite width propagated towards the surface will be compressed; its tail moves faster than its head. Naturally one may ask under which circumstances this gives rise to shock formation. In order to investigate this question, we consider model 1 of Table I.

First, however, we need to take care of a numerical difficulty arising in this context. In order to guarantee adequate numerical resolution of the expected features close to the surface, we switch from the radial coordinate $x$ to a rescaled radius $y$ related by

$$
d y=\frac{1}{\bar{C}} d \bar{r} \rightarrow \bar{r}_{, y}=\bar{C},
$$

where $\bar{C}^{2}=\frac{\partial \bar{P}}{\partial \bar{\rho}}$ is the speed of sound. A straightforward calculation shows that the speed of sound measured in terms of $y$ now approaches a finite value at the surface, thus eliminating the danger of under resolving features. ${ }^{3}$

\footnotetext{
${ }^{3}$ We note that this potential lack of numerical resolution is not a problem in the evolution of the eigenmodes discussed above because of their oscillatory character.
} 

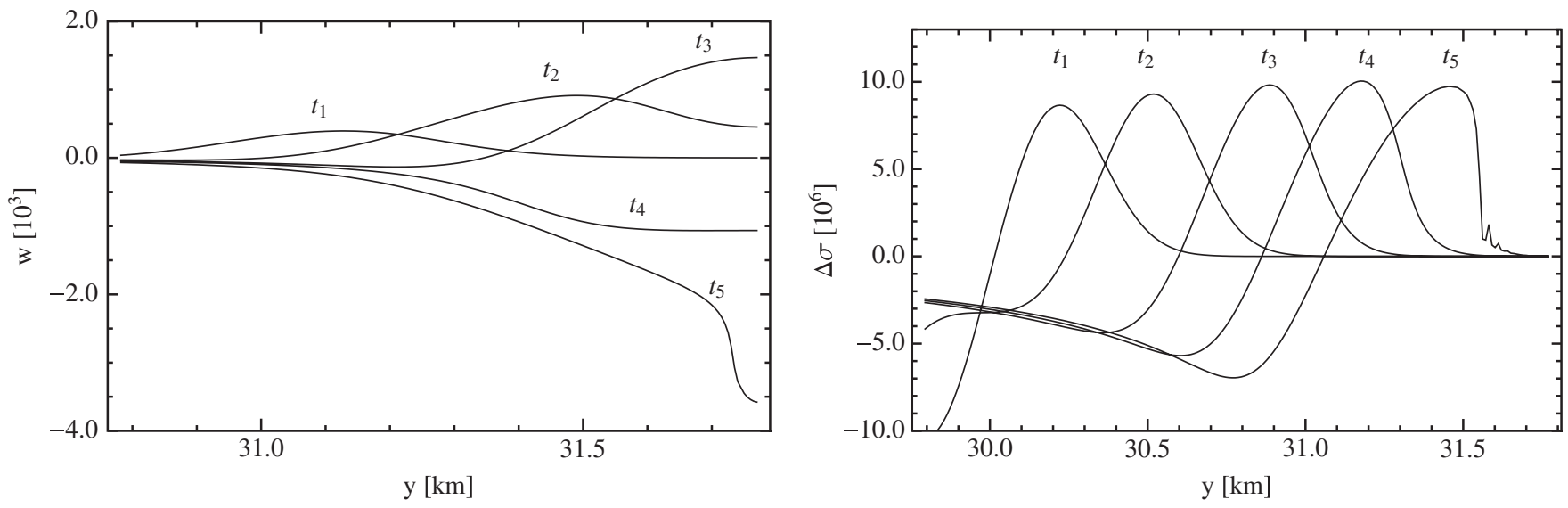

FIG. 10. Snapshots of the evolution of the velocity $w$ for a moderate amplitude $B=2 \times 10^{-4}$ of the initial perturbation (left panel) and of $\Delta \sigma$ obtained for an amplitude $B=10^{-3}$.

Even more remarkably, this decompactification is achieved by mapping a finite interval in $x$ to a finite interval in the new variable $y$. For more details on this procedure we refer the reader to $[62,63]$. For the numerical analysis we perturb model 1 by setting the initial velocity

$$
w(t=0)=B e^{-\left(\left(\left(y-y_{0}\right)^{2}\right) /(b)\right)},
$$

where width and center of the Gaussian pulse are set to $b=$ $0.05 \mathrm{~km}^{2}$ and $y_{0}=30 \mathrm{~km}$, respectively. The general behavior of this pulse during a fully nonlinear time evolution now depends exclusively on its initial amplitude $B$. In practice, we observe three different types of behavior. For sufficiently small amplitudes, the pulse is reflected at the surface and we do not observe shock formation. At moderate amplitudes the pulse gets reflected at the boundary, but a discontinuity in $w$ as well as $\Delta \sigma$ develops shortly after reflection. This is shown in the left panel of Fig. 10 for an amplitude $B=2 \times 10^{-4}$. As we further increase the amplitude, discontinuities develop in the variables $w, \Delta \lambda$ and $\Delta \sigma$ before the pulse reaches the stellar surface. This scenario is illustrated for the variable $\Delta \sigma$ and using an initial amplitude $B=10^{-3}$ in the right panel of Fig. 10 .

Shock formation at the stellar surface has recently been studied in the context of a plane parallel model in Newtonian gravity by Gundlach and Please [64]. In particular, their Eq. (27) provides the minimum amplitude required for shock formation in terms of amplitude $v_{0}$ as well as width $\tilde{\sigma}_{1}$ and initial location $\tilde{\sigma}_{0}$ of the initial pulse. (We use a tilde to distinguish their $\tilde{\sigma}$ from our evolution variable defined in Eq. (3).) This equation neglects a factor $1 / 2$, which we reintroduce for the comparison with our numerical simulations. The starting point for the following discussion is therefore

$$
\frac{v_{0}}{\tilde{\sigma}_{1}} \lesssim \frac{1}{2}\left(\frac{\tilde{\sigma}_{1}}{\tilde{\sigma}_{0}}\right)^{n+(1 / 2)},
$$

where the polytropic index is defined by $\gamma=1+1 / n$. Translating this expression into the coordinates used throughout this work, we arrive at the following condition ${ }^{4}$

$$
D \equiv \frac{v_{0} x_{\star}^{n+1}}{\bar{r}_{1}^{n+(3 / 2)}} \lesssim \sqrt{g}=\text { const. }
$$

Here $x_{\star}=\left|r_{\mathrm{S}}-\bar{r}_{0}\right|, \bar{r}_{0}$ is the position and $\bar{r}_{1}$ the characteristic length scale of the initial perturbation and $r_{\mathrm{S}}$ and $g$ are radius and gravitational acceleration at the surface of the background model, respectively. The derivation of relation (31) does not take into account the precise shape of the wave packet, however, which may enter as a dimensionless constant.

In order to test their prediction numerically we perform a series of simulations with Gaussian initial data. As a measure of the characteristic length scale $\bar{r}_{1}$ in Eq. (32), we choose the full width at half maximum (FWHM) of the Gaussian. For our model 1 we have a polytropic index $n=1$ and $\sqrt{g}=\sqrt{M / r_{\mathrm{S}}^{2}}=0.1337$. The resulting two-parameter study is summarized in Table IX. For each combination of width $\bar{r}_{1}$ and initial position $\bar{r}_{0}$ we start with a very small amplitude of the initial perturbation and repeat the simulation at increasingly larger amplitude until we observe formation of a discontinuity. The resulting threshold amplitude $v_{0}$ is then used to evaluate the left-hand side of Eq. (32).

The numerical uncertainties in this study are dominated by the difficulties in determining when a discontinuity has actually formed. First, we have to discretize the amplitude $v_{0}$ in order to keep the number of simulations at an acceptable level. Second, numerical dissipation may suppress shock formation. Finally, there is some freedom in choosing a critical gradient to define a discontinuity. In our simulations we choose the critical value to be $w_{, y}=$ $0.05 \mathrm{~km}^{-1}$. Bearing in mind all these difficulties, we estimate the numerical accuracy of the critical amplitude $v_{0}$ to

\footnotetext{
${ }^{4}$ This expression corrects Eq. (28) in [64] by adding a missing factor $\sqrt{x_{\star} / x_{0}}$.
} 
TABLE IX. The left-hand side of Eq. (32) for critical perturbations that are sufficiently large to generate shocks close to the surface.

\begin{tabular}{lcccc}
\hline \hline r in [km] & \multicolumn{4}{c}{$\frac{v_{0}\left|r_{\mathrm{S}}-\bar{r}_{0}\right|^{n+1}}{\bar{r}_{1}^{n+(3 / 2)}}$ for initial width $\sqrt{b / 2}$} \\
& 0.2 & 0.3 & 0.4 & 0.5 \\
\hline 6.79 & 0.180 & 0.196 & 0.204 & 0.207 \\
7.13 & 0.168 & 0.182 & 0.189 & 0.191 \\
7.45 & 0.157 & 0.170 & 0.176 & 0.178 \\
7.76 & 0.148 & 0.160 & 0.165 & 0.167 \\
8.05 & 0.141 & 0.152 & 0.157 & 0.158 \\
8.32 & 0.135 & 0.145 & 0.150 & 0.151 \\
8.58 & 0.130 & 0.140 & 0.144 & 0.145 \\
8.82 & 0.126 & 0.135 & 0.139 & 0.140 \\
9.05 & 0.123 & 0.132 & 0.135 & 0.136 \\
9.27 & 0.120 & 0.129 & 0.132 & 0.133 \\
9.47 & 0.118 & 0.126 & 0.130 & 0.130 \\
9.66 & 0.117 & 0.125 & 0.128 & 0.129 \\
9.83 & 0.115 & 0.123 & 0.127 & 0.128 \\
9.99 & 0.114 & 0.123 & 0.126 & 0.127 \\
10.13 & 0.114 & 0.122 & 0.126 & 0.128 \\
10.27 & 0.114 & 0.122 & 0.126 & 0.128 \\
10.38 & 0.114 & 0.123 & 0.127 & 0.130 \\
10.46 & 0.114 & 0.123 & 0.129 & 0.131 \\
10.58 & $(0.115)$ & $(0.125)$ & $(0.131)$ & $(0.134)$ \\
10.65 & $(0.116)$ & $(0.127)$ & $(0.134)$ & $(0.139)$ \\
10.72 & $(0.118)$ & $(0.130)$ & $(0.138)$ & $(0.141)$ \\
\hline \hline
\end{tabular}

be about $10 \%$. Within these uncertainties, we observe good agreement with the predictions of [64]. The obtained values for $D$ are listed in Table IX.

As long as the initial position of the pulse is not too close to the center the left-hand side of Eq. (32) shows little variation with the pulse width and initial location. Bearing in mind that (32) has been derived for plane parallel geometry and in the Newtonian approximation, the observed increase in variability of the coefficient in Table IX at small $x_{0}$ is not surprising. For radii close to the surface the determination of the width and the position of the pulse is limited because the pulse steepens and deviates significantly from a purely Gaussian profile. Values obtained in this regime are thus given in parentheses.

In summary, Eq. (32) as well as the numerical study illustrate nicely that the significance of nonlinear effects does not only depend on the amplitude of the evolving feature. Instead, the length scale on which profiles change is equally important and may generate significant deviations from perturbative predictions at comparatively small amplitudes of the signal.

For completeness we note that the stellar model used in this study oversimplifies the structure of the stellar surface. A crust expected to form at the surface of a neutron star is likely to result in more complex phenomena and requires a more detailed treatment. As has been mentioned above, however, the primary purpose of our study is to probe the taxonomy of nonlinear effects in the weakly and moderately nonlinear regime. We postpone the sophistication of a realistic neutron star model to future work. In this spirit, our analysis leads to two main conclusions. First, the theoretical modeling of neutron star surfaces requires particular care and, second, the surface exhibits a rich phenomenology, not always concurrent with immediately intuitive expectations.

\section{SUMMARY}

In this paper we have investigated a variety of nonlinear effects associated with radial oscillations of polytropic neutron star models. We have performed our study in the framework of fully nonlinear general relativity and have achieved a high level of numerical precision by formulating the time evolution in terms of finite deviations from an equilibrium configuration which in our case is given by a TOV model.

The first nonlinear effect studied in detail is the coupling of eigenmodes due to nonlinear effects. In the absence of a unique decomposition of a fully nonlinear dynamic model into background and deviations, we have performed oneparameter studies using as initial data the equilibrium configuration perturbed in the form of one single eigenmode profile with varying amplitude. Our results and their interpretation are to be understood in the context in this particular construction of a reference background. By virtue of the completeness of the eigenmode spectrum, we are able to measure the excitation of modes not contained in the initial data by calculating eigenmode coefficients from overlap integrals. This analysis has revealed two qualitatively different regimes depending on the amplitude of the initial data. For sufficiently low values, we find the excitation of modes to increase quadratically with the amplitude. In the context of analytic studies based on coupled oscillators, as, for example, employed by Van Hoolst [22], we interpret this quadratic dependence as a leading-order coupling between different modes. In the case of an initial perturbation given by the fundamental eigenmode, this quadratic dependence persists over the entire range of initial surface displacements of up to $70 \mathrm{~m}$. Initial data of higher modes with sufficient amplitude, however, result in a transition in the excitation of modes to higher-order power laws. Specifically, our results indicate that secondarily excited modes appear in multiplets; initial data of eigenmode $j$ excites modes $i=j+1, \ldots, 2 j$ according to a quadratic power law, modes $i=2 j+1, \ldots, 3 j$ with cubic dependence and so on. Strong resonance effects may cause some complications, however, which manifest themselves in deviations from this rule. This behavior demonstrates the significance of higher-order coupling of eigenmodes. The onset of this moderately nonlinear regime occurs at amplitudes of the order of a few meters, but the details appear to vary with the stellar model. As a general rule, we find higher-order modes to pass energy 
to lower-order modes more efficiently than the other way around.

Our results confirm the intuitive expectation that eigenmodes with an integer frequency ratio interact particularly efficiently. This resonance manifests itself most conspicuously in the second and fourth eigenmode of the stiffer model considered in this study where the frequency ratio is equal to two within three digits. Initial data in the form of the second mode can excite the fourth mode with such efficiency that both amplitudes become comparable. When this happens, we observe a further complication in the nonlinear behavior: the strong excitation of mode 4 results in a significant transfer of energy to other eigenmodes and its amplitude saturates, i.e. it stops growing in accordance with the expected power law. A similar behavior is observed for other pairs of modes, as, for example, modes 2 and 6. Overall, resonance appears to be weaker for larger deviations of the involved frequency ratios from an integer value.

A particularly fertile ground for the analysis of nonlinear effects is given by stellar models close to the stability limit of the mass-radius relation. Instability manifests itself in the vanishing of the oscillation frequency of the fundamental eigenmode [7] in which case the linear (in the deviations) terms in the evolution equations cancel. The resulting dominance of higher-order terms leads to a variety of effects. Most notably, our results show that nonlinear effects have the capacity to stabilize stars expected to be unstable in linear analysis; initial perturbations lead to a periodic pulsation of the star instead of the expected gravitational collapse. Nonlinearity further manifests itself in a visible distortion of the time dependence of the oscillations away from sinusoidal character. Also, the oscillation pattern depends on the initial phase of the perturbation. It will be interesting to investigate in future work to what extent this stabilization is a generic feature and how it depends on the physical properties of the stellar model.

Finally, we have studied the formation of discontinuities near the surface of the star by evolving Gaussian pulses propagating from the stellar interior towards the surface. In particular, we have compared our numerical findings with analytic predictions by Gundlach and Please [64] who give threshold amplitudes for the initial amplitude of the pulse depending on its initial location in the sense that amplitudes above this threshold lead to shock formation and those below do not. Within the accuracy of the analysis, approximately $10 \%$, our numerical results confirm the predictions of Gundlach and Please in the range of validity of their model.

In summary, the precision of the numerical method proposed here has enabled us to identify a number of nonlinear effects with no approximation other than that of a rather simple stellar model. Our results suggest a variety of extensions for future work. These include the refinement of the stellar model by including, for example, realistic equations of state, magnetic fields and, most importantly, relaxing the condition of spherical symmetry. The main obstacle for the latter appears to us to be the extension of the comoving, Lagrangian formulation to higher dimensional problems. We find a careful treatment of the stellar surface to be crucial in our study. A possible avenue towards achieving this goal is the use of level set methods and fast marching methods [65] specifically designed to follow the motion of interfaces in numerical simulations.

\section{ACKNOWLEDGMENTS}

We thank Philippos Papadopoulos for various fruitful discussions. This work was supported by DFG grant SFB/ Transregio 7 "Gravitational Wave Astronomy," by grants from the Sherman Fairchild Foundation to Caltech and by NSF Grant Nos. PHY-0601459 and PHY-0652995. U.S. acknowledges support from NSF TeraGrid Grant No. PHY090003. N. A. acknowledges support from STFC in the United Kingdom via Grant No. PP/E001025/1.

\section{APPENDIX A: THE EIGENMODE EQUATION}

In the limit of infinitesimally small deviations $\Delta f$ from the equilibrium variables $\bar{f}$, the time evolution of the star is governed by the linearized version of the system (14)-(18). The combination of these equations into the single, secondorder partial differential Eq. (19) for the rescaled displacement $\zeta$ is discussed in detail in [60]. The coefficients $W, \Pi$ and $Q$ in that equation are given by

$$
\begin{gathered}
\Pi=\bar{C}^{2}(\bar{\rho}+\bar{P}) \frac{\bar{\lambda}^{3} \bar{\mu}}{\bar{r}^{2}} \\
W=(\bar{\rho}+\bar{P}) \frac{\bar{\lambda} \bar{\mu}^{3}}{\bar{r}^{2}} \\
Q=\frac{\Pi}{\bar{C}^{2}}\left[\left(\frac{\bar{\lambda}_{, x}}{\bar{\lambda}}\right)^{2}+\frac{4}{\bar{r}} \frac{\bar{\lambda}_{, x}}{\bar{\lambda}}-8 \pi \bar{\mu}^{2} \bar{P}\right],
\end{gathered}
$$

where the metric function $\bar{\mu}=(1-2 \bar{N} \bar{r})^{-1 / 2}$.

In order to numerically solve the eigenmode equation, we write it as a first-order system in the variable $\xi$ and make the ansatz $\xi(t, x)=\xi(x) e^{i \omega t}$.

$$
0=\bar{C}^{2} \xi_{, x}-B
$$

$$
\begin{gathered}
B_{, x}=-\frac{\bar{C}^{2} \bar{r}_{, x}}{\Pi}\left\{\frac{\bar{\lambda}}{\bar{r}^{2}}\left[\frac{\Pi}{\bar{r}_{, x}}\left(\frac{\bar{r}^{2}}{\bar{\lambda}}\right)_{, x}\right]_{, x}+\bar{r}_{, x}\left(\omega^{2} W+Q\right)\right\} \xi \\
-\left\{\frac{\bar{r}_{, x}}{\Pi}\left(\frac{\bar{\lambda}}{\bar{r}^{2}}\right)^{2}\left[\left(\frac{\bar{r}^{2}}{\bar{\lambda}}\right)^{2} \frac{\Pi}{\bar{r}_{, x}}\right]_{, x}-\frac{\bar{C}_{, x}^{2}}{\bar{C}^{2}}\right\} B \\
0=\left(\omega^{2}\right)_{, x} .
\end{gathered}
$$

The last equation simply expresses the fact that the frequency $\omega$ is constant. The purpose of introducing this 
equation becomes apparent when we consider the boundary conditions. Spherical symmetry requires the displacement $\xi$ to vanish at the origin. The auxiliary variable $B$ vanishes by definition at the stellar surface. The value of $B$ at the origin determines the amplitude of the profile $\xi(x)$. Because eigenmodes are only defined up to a constant factor, we are free to choose for $B(0)$ an arbitrary finite value. We thus have a two-point-boundary-value problem for the three variables $\xi(x), B(x)$ and $\omega(x)$ which we solve with a relaxation algorithm. In particular, the solution for the constant $\omega(x)$ provides us with the frequency of the eigenmode. Solutions only exist for specific values of the frequency and finding a given mode requires a relatively careful initial guess.
[1] H.S. Leavitt and E.C. Pickering, Harvard College Observatory Circular 173, 1 (1912).

[2] E. C. Pickering, Harvard College Observatory Circular 54, 1 (1901).

[3] C. Alcock et al., Astron. J. 109, 1653 (1995).

[4] G. F. Benedict et al., Astron. J. 133, 1810 (2007).

[5] A. Olech and P. Moskalik, arXiv:0812.4173.

[6] A. C. Birch, Journal of Physics Conference Series 118, 012009 (2008).

[7] S. Chandrasekhar, Phys. Rev. Lett. 12, 114 (1964).

[8] B.S. Sathyaprakash and B.F. Schutz, Living Rev. Relativity 12 (2009), http://relativity.livingreviews.org/ Articles/lrr-2009-2/download/index.html.

[9] G. Chanmugam, Astrophys. J. 217, 799 (1977).

[10] E. N. Glass and L. Lindblom, Astrophys. J. Suppl. Ser. 53, 93 (1983).

[11] K. D. Kokkotas and J. Ruoff, Astron. Astrophys. 366, 565 (2001).

[12] H. M. Vaeth and G. Chanmugam, Astron. Astrophys. 260, 250 (1992).

[13] Z. Kolláth, J.P. Beaulieu, J. R. Buchler, and P. Yecko, Astrophys. J. 502, L55 (1998).

[14] A. K. Schenk, P. Arras, É.É. Flanagan, S. A. Teukolsky, and I. Wasserman, Phys. Rev. D 65, 024001 (2002).

[15] P. Arras, E. E. Flanagan, S. M. Morsink, A. K. Schenk, S. A. Teukolsky, and I. Wasserman, Astrophys. J. 591, 1129 (2003).

[16] R. Bondarescu, S.A. Teukolsky, and I. Wasserman, arXiv:0809.3448.

[17] J. Brink, S. A. Teukolsky, and I. Wasserman, Phys. Rev. D 70, 124017 (2004).

[18] J. Brink, S. A. Teukolsky, and I. Wasserman, Phys. Rev. D 71, 064029 (2005).

[19] L. Lin and W. Suen, Mon. Not. R. Astron. Soc. 370, 1295 (2006).

[20] W. Dziembowski, Acta Astronomica 32, 147 (1982).

[21] P. Kumar and P. Goldreich, Astrophys. J. 342, 558 (1989).

[22] T. Van Hoolst, Astron. Astrophys. 308, 66 (1996).

[23] A. Passamonti, M. Bruni, L. Gualtieri, A. Nagar, and C. F. Sopuerta, Phys. Rev. D 73, 084010 (2006).

[24] A. Passamonti, N. Stergioulas, and A. Nagar, Phys. Rev. D 75, 084038 (2007).

[25] D. Brizuela, J.M. Martín-García, and G. A. MenaMarugan, Phys. Rev. D 74, 044039 (2006).

[26] D. Brizuela, J.M. Martín-García, and G. A. MenaMarugan, Phys. Rev. D 76, 024004 (2007).

[27] D. Brizuela, J. M. Martín-García, U. Sperhake, and K.
Kokkotas, High-order perturbations of a spherical collapsing star (forthcoming).

[28] D. Brizuela, J. M. Martín-García, and M. Tiglio, Phys. Rev. D 80, 024021 (2009).

[29] E. Berti, V. Cardoso, and C. M. Will, Phys. Rev. D 73, 064030 (2006).

[30] V. Ferrari and L. Gualtieri, Gen. Relativ. Gravit. 40, 945 (2008).

[31] E. Berti, V. Cardoso, J. A. González, U. Sperhake, M. D. Hannam, S. Husa, and B. Brügmann, Phys. Rev. D 76, 064034 (2007).

[32] K. Kokkotas, Living Rev. Relativity 2 (1999), http:// relativity.livingreviews.org/Articles/lrr-1999-2/download/ index.html.

[33] E. W. Leaver, Phys. Rev. D 34, 384 (1986).

[34] H. Nakano and K. Ioka, Phys. Rev. D 76, 084007 (2007).

[35] J. G. Baker, W. D. Boggs, J. Centrella, B. J. Kelly, S. T. McWilliams, and J. R. van Meter, Phys. Rev. D 78, 044046 (2008).

[36] E. Berti, V. Cardoso, J. A. González, and U. Sperhake, Phys. Rev. D 75, 124017 (2007).

[37] A. Buonanno, G. B. Cook, and F. Pretorius, Phys. Rev. D 75, 124018 (2007).

[38] P. Papadopoulos and C.F. Sopuerta, Phys. Rev. D 65, 044008 (2002).

[39] H. Dimmelmeier, N. Stergioulas, and J. A. Font, Mon. Not. R. Astron. Soc. 368, 1609 (2006).

[40] J. Font, H. Dimmelmeier, A. Gupta, and N. Stergioulas, Mon. Not. R. Astron. Soc. 325, 1463 (2001).

[41] L. Lindblom, J. E. Tohline, and M. Vallisneri, Phys. Rev. Lett. 86, 1152 (2001).

[42] L. Lindblom, J. E. Tohline, and M. Vallisneri, Phys. Rev. Lett. 86, 1152 (2001).

[43] N. Stergioulas and J. A. Font, Phys. Rev. Lett. 86, 1148 (2001).

[44] N. Stergioulas, T. A. Apostolatos, and J. A. Font, Mon. Not. R. Astron. Soc. 352, 1089 (2004).

[45] M. Anderson, E. W. Hirschmann, L. Lehner, S. L. Liebling, P. M. Motl, D. Neilsen, C. Palenzuela, and J. E. Tohline, Phys. Rev. D 77, 024006 (2008).

[46] L. Baiotti, B. Giacomazzo, and L. Rezzolla, Phys. Rev. D 78, 084033 (2008).

[47] L. Baiotti et al., Phys. Rev. D 71, 024035 (2005).

[48] M. D. Duez, F. Foucart, L. E. Kidder, H. P. Pfeiffer, M. A. Scheel, and S. A. Teukolsky, Phys. Rev. D 78, 104015 (2008).

[49] M. D. Duez, Y. T. Liu, S. L. Shapiro, M. Shibata, and B. C. 
Stephens, Phys. Rev. D 73, 104015 (2006).

[50] J. A. Font, T. Goodale, S. Iyer, M. Miller, L. Rezzolla, E. Seidel, N. Stergioulas, W. M. Suen, and M. Tobias, Phys. Rev. D 65, 084024 (2002).

[51] P. Marronetti, M.D. Duez, S.L. Shapiro, and T.W. Baumgarte, Phys. Rev. Lett. 92, 141101 (2004).

[52] M. Miller, P. Gressman, and W.-M. Suen, Phys. Rev. D 69, 064026 (2004).

[53] M. Shibata and K. Taniguchi, Phys. Rev. D 73, 064027 (2006).

[54] U. Sperhake, P. Papadopoulos, and N. Andersson, arXiv: astro-ph/0110487.

[55] M. May and R. White, Phys. Rev. 141, 1232 (1966).

[56] C. Misner and D. Sharp, Phys. Rev. 136, B571 (1964).

[57] E. Seidel, Phys. Rev. D 42, 1884 (1990).

[58] J. Oppenheimer and G. Volkoff, Phys. Rev. 55, 374 (1939).
[59] R. Tolman, Phys. Rev. 55, 364 (1939).

[60] C. Misner, K. Thorne, and J. Wheeler, Gravitation (W. H. Freeman, New York, 1973).

[61] W. Press, S. Teukosky, W. Vetterling, and B. Flannery, Numerical Recipes in C (Cambridge University Press, Cambridge, 1992), 2nd ed..

[62] U. Sperhake, Ph.D. thesis, University of Southampton, 2001.

[63] J. Ruoff, Ph.D. thesis, Fakultät für Physik der EberhardKarls-Universität Tübingen, 2000.

[64] C. Gundlach and C. Please, Phys. Rev. D 79, 067501 (2009).

[65] J.A. Sethian, Level Set Methods and Fast Marching Methods (Cambridge University Press, Cambridge, England, 1999), 2nd ed.. 